\title{
O VALOR DA RESSONÂNCIA MAGNÉTICA NA DETECC̣̃̃o DE NÓDULOS HEPÁTICOS UTILIZANDO-SE DIVERSAS TÉCNICAS PONDERADAS EM T2: EXISTE CONSENSO?*
}

\author{
Glaucia Andrade e Silva Palácio ${ }^{1}$, Cristiane Lima Abbehusen ${ }^{1}$, Dario Ariel Tiferes ${ }^{2}$, Giuseppe \\ D'Ippolito $^{3}$, Jacob Szejnfeld ${ }^{4}$
}

Resumo Desde o início da década de 80 a ressonância magnética vem sendo utilizada para o estudo do abdome e principalmente na detecção de nódulos hepáticos. As imagens ponderadas em T2 são as que trouxeram maior benefício quando comparadas à tomografia computadorizada com contraste. Inúmeras técnicas e seqüências de ressonância magnética ponderadas em T2 surgiram desde então, na tentativa de aumentar a eficácia diagnóstica, com menores tempos de exame. Neste sentido, foram publicados inúmeros trabalhos demonstrando a utilidade de seqüências rápidas e ultra-rápidas, com e sem supressão de gordura, em apnéia, com sincronizador respiratório e com bobinas de sinergia, entre outros avanços tecnológicos. No entanto, não há um consenso sobre qual a técnica mais apropriada e sensível para a detecção de lesões hepáticas focais. Neste artigo fazemos uma revisão bibliográfica e análise crítica das diversas técnicas de imagens ponderadas em T2, no que diz respeito às suas sensibilidades na detecção de nódulos hepáticos.

Unitermos: Ressonância magnética; Seqüências de pulso; Tumores hepáticos; Fígado.

Abstract The value of magnetic resonance imaging for the detection of focal liver lesions using different T-2 weighted techniques: is there a consensus?

Since the early 1980's several magnetic resonance imaging pulse sequences have been developed in order to determine the optimum imaging technique for the detection and characterization of hepatic lesions. T2weighted images play an important role in the evaluation of the liver and present equal or greater sensitivity than enhanced computed tomography for the detection of liver lesions. New techniques for obtaining T-2 weighted images have been developed in the attempt to optimize the method. These techniques have improved the image quality by shortening examination time, reducing motion artifacts, and improving contrast-to-noise ratio. The effectiveness of the different techniques (fat suppression, breath-hold, respiratorytriggered and phased-array coils) has been tested in many comparative studies, although the results are controversial. In this article we review the literature and discuss the several T2-weighted image techniques, particularly with regard to sensitivity to detect focal liver lesions.

Key words: Magnetic resonance imaging; Pulse sequences; Liver tumors; Liver.

\section{INTRODUÇÃO}

A ressonância magnética $(\mathrm{RM})$ é um método muito sensível na detecção de nódulos hepáticos. Além de proporcionar detalhada informação anatômica, permite obter imagens em múltiplos planos e com alto

\footnotetext{
* Trabalho realizado no Departamento de Diagnóstico por Imagem da Universidade Federal de São Paulo - Escola Paulista de Medicina (Unifesp-EPM), São Paulo, SP.

1. Pós-graduandas do Departamento de Diagnóstico por Imagem da Unifesp-EPM.

2. Médico do Departamento de Diagnóstico por Imagem da Unifesp-EPM.

3. Professor Adjunto do Departamento de Diagnóstico por Imagem da Unifesp-EPM

4. Chefe do Departamento de Diagnóstico por Imagem da Unifesp-EPM.

Endereço para correspondência: Dra. Glaucia Andrade e Silva Palácio. Rua Napoleão de Barros, 920, apto. 61 São Paulo, SP, 04024-002. E-mail: gl.palacio@bol.com.br

Recebido para publicação em 10/1/2002. Aceito, após revisão, em $3 / 5 / 2002$.
}

contraste entre os diferentes tecidos. Nas últimas décadas, muitos protocolos foram desenvolvidos para o exame de RM do fígado, utilizando diversas técnicas de aquisição de imagem. Estes protocolos variam amplamente quanto à escolha das sequiências de pulso e dos parâmetros técnicos utilizados. A busca pela sequiência ideal, ou seja, aquela com maior eficácia na detecção de lesões focais, com elevada resolução espacial e baixa resolução temporal, e insensível a artefatos, tem sido motivo de inúmeros estudos. Apesar dos recentes avanços na aquisição de imagens ponderadas em T1, com técnica dinâmica (gradiente-eco) e injeção de contraste, as sequiências ponderadas em $\mathrm{T} 2$ têm-se mostrado indispensáveis, por sua elevada capacidade na detecção e caracterização de lesões hepáticas focais ${ }^{(\mathbf{1})}$. Porém, ainda não existe um consenso, principalmente quanto ao uso de técnicas com supressão de gordura, sincronizador respiratório e sequiências em apnéia, particularmente úteis na obtenção de imagens ponderadas em $\mathrm{T} 2$.

Este artigo tem como objetivo realizar uma revisão bibliográfica racional e análise crítica das diversas técnicas de imagens ponderadas em $\mathrm{T} 2$, no que diz respeito principalmente à sua sensibilidade na detecção de lesões hepáticas focais.

\section{REVISÃO DA LITERATURA}

\section{Seqüiências ponderadas em T2}

As primeiras imagens de RM com utilidade clínica foram realizadas no começo dos anos 80, sendo o cérebro o primeiro órgão de interesse. Os longos tempos de aquisição das seqüências prejudicavam seu 
uso no estudo do abdome, devido à presença de muitos artefatos de movimento ${ }^{(2)}$.

O rápido desenvolvimento das técnicas e equipamentos limitou a questão dos artefatos, e hoje em dia a RM é um dos principais métodos de imagem para a avaliação do abdome, e particularmente do fígado.

Já nos primeiros trabalhos que avaliaram a capacidade da RM em detectar lesões hepáticas focais ressaltava-se a importância da seleção adequada de seqüências de pulso a serem utilizadas ${ }^{(\mathbf{3})}$.

A primeira seqüência ponderada em $\mathrm{T} 2$ utilizada clinicamente para o estudo do fígado foi a spin-eco (SE), com bons resultados quanto à detecção e caracterização de lesões hepáticas focais. No entanto, os seus longos tempos de aquisição (de até 15 minutos) e, portanto, maior propensão a artefatos de movimento, fez com que fosse progressivamente substituída por técnicas mais rápidas ${ }^{(\mathbf{4 , 5})}$.

No início da década de 90 surgiram as primeiras sequiências rápidas, conhecidas como turbo-SE (TSE) ou "fast"-SE (FSE), dependendo do fabricante, que permitiram reduzir de forma significativa os tempos de aquisição, principalmente das imagens ponderadas em T2. Este tipo de seqüência consiste na aplicação de um pulso inicial de $90^{\circ}$, seguido de um número de pulsos de refocalização de $180^{\circ}$. Após cada um destes pulsos de refocalização, um eco é medido. Isto cria grupos com um número correspondente de ecos com diferentes codificações de fase. Os grupos são adquiridos em intervalos regulares (TR) até a imagem ser completada ${ }^{(\mathbf{6})}$.

A partir de 1993, trabalhos comparando as seqüências TSE e SE ponderadas em T2 foram realizados. Low et al. (1993) referiram sensibilidade discretamente superior da sequiência TSE quando comparada com a SE na detecção de lesões hepáticas focais, em 26 pacientes $^{(\mathbf{6})}$. Estudos posteriores confirmaram a vantagem da utilização da seqüência TSE, que apresentou sensibilidade na detecção de nódulos hepáticos semelhante ou superior à seqüência SE convencional, e com tempo de exame significantemente inferior ${ }^{(7-15)}$.

Com o aprimoramento dessas seqüências rápidas (TSE, FSE) e sua realização em menores intervalos de tempo, minimizava-se uma das principais limitações, mas os artefatos de movimento respiratório ainda eram freqüentes. Quando se utilizava sincronizador respiratório a qualidade da imagem melhorava, porém à custa de um tempo relativamente longo de aquisição (três a cinco minutos). Neste momento, a melhora dos equipamentos já permitia a realização dessas sequiências em apnéia.

Em 1995, Rydberg et al. avaliaram, prospectivamente, 31 pacientes com lesões hepáticas malignas e compararam as seqüências FSE com e sem apnéia, e SE convencional. Todas as seqüências foram realizadas com saturação de gordura e bobina de sinergia ("phased-array"). A seqüência SE foi a que subjetivamente melhor demonstrou as lesões, apesar de não ter havido diferença estatisticamente significante no número de lesões detectadas ${ }^{(\mathbf{1 6})}$. No mesmo ano, Helmberger et al. também obtiveram sensibilidades semelhantes na detecção de lesões focais utilizando seqüências FSE em apnéia e $\mathrm{SE}^{(\mathbf{1 7 )}}$.

Nos últimos anos, novas seqüências ultra-rápidas foram desenvolvidas, como a "half-Fourier aquisition single-shot turbo spin echo" (HASTE) ou a "single-shot fast spin echo" (SSFSE), com a capacidade de adquirir imagens ponderadas em T2 em poucos segundos, sendo muito útil em pacientes com dificuldade de manter apnéia.

Embora alguns autores acreditem que a seqüência HASTE possa substituir a seqüência SE convencional e até mesmo a seqüência FSE na avaliação de lesões focais hepáticas ${ }^{(\mathbf{1 5 , 1 8 - 2 5})}$, outros referiram acurácia significantemente superior da seqüência FSE sobre a HASTE na detecção de lesões hepáticas sólidas e císticas ${ }^{(\mathbf{2 6})}$.

A seqüência eco-planar (EPI) ponderada em T2 foi desenvolvida para realizar imagens ultra-rápidas (de 4 a 18 segundos) e tornou-se disponível após a introdução de equipamentos com sistemas de gradien-

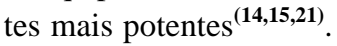

Estudos iniciais, utilizando seqüência EPI do tipo "single-shot" (com tempos de aquisição de quatro segundos) mostraram sensibilidade inferior às seqüências HASTE e TSE na detecção de lesões focais e baixa resolução espacial ${ }^{(\mathbf{1 4 , 1 5 , 2 1 )}}$. Trabalhos mais recentes, utilizando seqüência EPI "multi-shot" (com tempo de aquisição em torno de 18 segundos) ${ }^{(27,28)}$, apresentaram resultados superponíveis à sequiência FSE, apesar de a prática ter demonstrado que a aplicação da seqüência eco-planar ainda está ligada a uma grande quantidade de artefatos e baixa relação sinal/ruído.

A seqüência GRASE combina gradiente-eco com spin-eco e é capaz de adquirir imagens de abdome ponderadas em T2 em tempos rápidos (cerca de 18 a 90 segundos) ${ }^{\text {(29-32) }}$. Alguns trabalhos demonstraram sensibilidade na detecção de lesões focais hepáticas semelhante ${ }^{(\mathbf{3 0 , 3 1})}$ e por vezes superior $^{(32)}$ à seqüência FSE.

Parece haver, na literatura, tendência em considerar as seqüências rápidas TSE ou FSE como as sequiências ponderadas em T2 mais sensíveis e apropriadas para o estudo de nódulos hepáticos. Novas sequiências ultra-rápidas, como HASTE, EPI e GRASE, têm sido estudadas e se mostrado promissoras, devido a uma ótima relação tempo de exame/eficácia diagnóstica.

\section{Sincronizador respiratório e apnéia}

Os artefatos de movimento são os mais freqüentes nos exames de RM do abdome superior e podem degradar significantemente a qualidade da imagem, diminuindo a sensibilidade da detecção de lesões. Dentre os vários artefatos de movimento, destacam-se os respiratórios.

Os artefatos de respiração podem ser reduzidos com a utilização de sincronização respiratória (SR), que consiste em uma técnica na qual o ciclo respiratório controla a coleta de dados da RM, ou diminuindo-se o tempo de aquisição a poucos segundos, o que permite adquirir as imagens durante uma única apnéia ${ }^{(33)}$.

Vários autores estudaram a sensibilidade na detecção de lesões hepáticas focais usando técnicas com SR e em apnéia.

Low et al. (1997), em estudo de 33 pacientes, obtiveram maior sensibilidade na avaliação de lesões hepáticas focais ao utilizar SR na seqüência FSE, comparada à mesma seqüência sem $\mathrm{SR}^{(\mathbf{3 4})}$.

Também em 1997, Tang et al. compararam seqüências TSE em apnéia e com SR, ambas com bobina de sinergia, em 58 pacientes com lesões hepáticas focais, e observaram que a sensibilidade, especificidade e acurácia na detecção das lesões foram maiores na seqüência realizada com $\mathrm{SR}^{(19)}$. Resultados semelhantes foram obtidos em outras séries ${ }^{(35-39)}$ (Figura 1). 

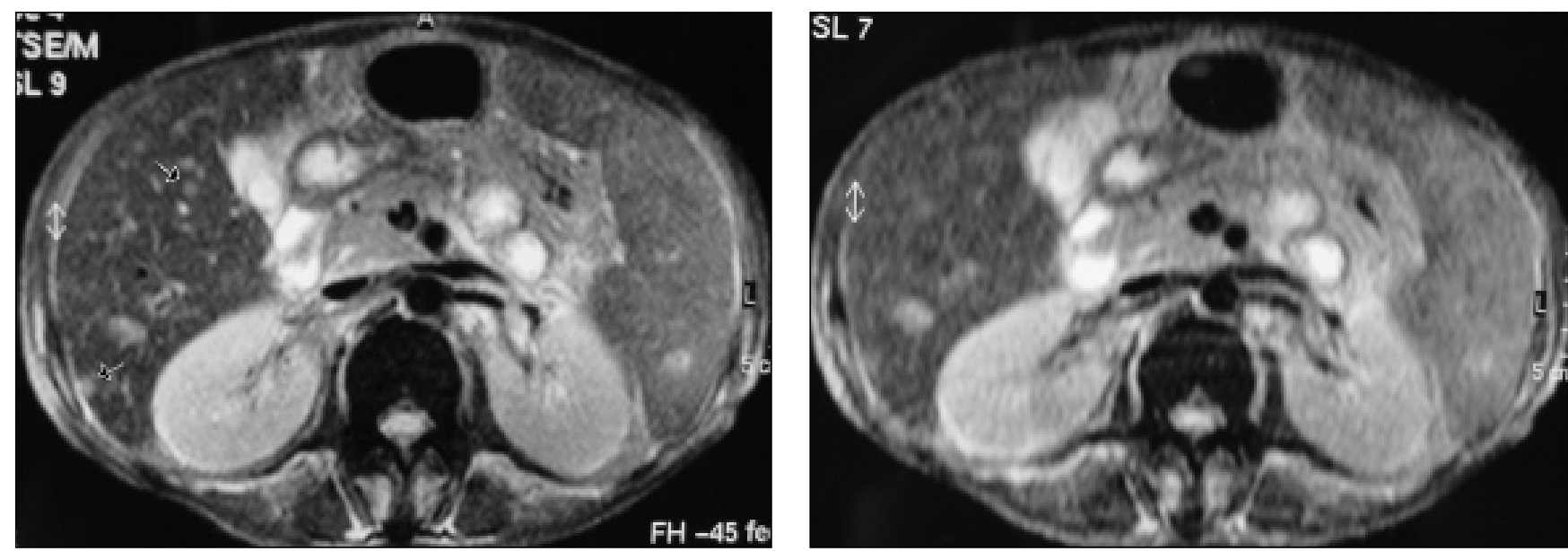

A

B

Figura 1. Seqüências TSE T2 (TR = 1.800, TE = 90) obtidas com supressão de gordura. A seqüência realizada com sincronizador respiratório (A) demonstrou duas lesões a mais que a seqüência realizada em apnéia $(\mathbf{B})$.

Carpenter et al. não verificaram diferença estatisticamente significante na sensibilidade de detecção de lesões hepáticas utilizando seqüências RARE (FSE) com e sem apnéia, em 19 pacientes com 106 lesões ${ }^{(\mathbf{4 0})}$. Resultados semelhantes foram observados por Soyer et al. ${ }^{\mathbf{( 4 1 , 4 2 )}}$. Outros autores, no entanto, referiram maior definição de margens (conspicuidade) de nódulos hepáticos com técnica FSE em apnéia quando comparado com imagens adquiridas sem apnéia ${ }^{(\mathbf{4 3 , 4 4 )}}$.

\section{Técnicas de supressão de gordura}

Existem várias vantagens na aplicação de técnicas de supressão de gordura nas imagens ponderadas em T2. A supressão de gordura reduz os artefatos de movimento do subcutâneo e da gordura intra-abdominal, aumenta a diferenciação do sinal entre os diferentes tecidos e melhora as relações sinal-ruído e contraste-ruído ${ }^{(45)}$.

Inicialmente, a supressão de gordura foi obtida utilizando a técnica "short time inversion recovery" (STIR). Esta técnica tem a vantagem de poder ser utilizada em equipamento com gradiente de baixa potência e suprimir uniformemente o sinal de toda a gordura, incluindo a sua fração aquosa. Porém, apresenta algumas desvantagens, como menor relação sinal/ruído e supressão de outros tecidos com T1 curto, como sangue, líquidos com elevado conteúdo protéico, melanina, e o gadolínio ${ }^{(45)}$.

Bydder et al., em 1985, observaram que a técnica STIR produzia imagens com alto contraste e mínima degradação por artefatos de movimento, sendo útil no rastreamento de metástases hepáticas ${ }^{(\mathbf{4 6})}$. A comparação de seqüências com e sem STIR por outros autores não revelou, contudo, diferenças significantes na sensibilidade de detecção de nódulos hepáticos ${ }^{\text {(22,24) }}$.

Outra técnica desenvolvida para a supressão de gordura foi a fat-sat ("fat saturation") ou SPIR ("spectral presaturation with inversion recovery”), que é específica para lipídios.

Schwartz et al. (1993) compararam, subjetivamente, as seqüências FSE com e sem supressão de gordura (fat-sat), e seqüência SE convencional na detecção de lesões focais. Os autores elegeram a seqüência FSE com supressão de gordura como a melhor para a detecção de nódulos hepáticos em $64 \%$ das vezes, contra $23 \%$ para a seqüência FSE sem supressão de gordura, e $13 \%$ para a $\mathrm{SE}^{(47)}$.

Vários trabalhos ${ }^{(26,48,49)}$ mostraram superioridade de imagens com supressão de gordura (utilizando diversas técnicas) na detecção de lesões hepáticas focais. Outros autores, no entanto, não encontraram diferenças estatisticamente significantes ${ }^{(\mathbf{2 3}, \mathbf{4 1}, \mathbf{4 2})}$ (Figura 2).

\section{Bobinas de sinergia}

A bobina de sinergia ("phased-array multicoils") é uma bobina de superfície constituída de um arranjo de pares de bobinas em quadratura destinadas à recepção simultânea de diferentes sinais de RM.
Com ela é possível obter imagens do abdome e pelve com elevada relação sinal/ ruído quando comparada à bobina de corpo convencional, que é integrada ao equipamento de RM. O aumento da relação sinal/ruído permite obter cortes mais finos, menores campos de visão e melhor resolução de imagem. Desta forma, teoricamente, um maior número de lesões poderia ser detectado. Uma desvantagem é seu elevado custo, que seria justificado se realmente aumentasse a sensibilidade na detecção de lesões hepáticas focais. Outra desvantagem é a presença de artefatos fantasmas nas seqüências sem supressão de gordura, devido ao intenso sinal do subcutâneo subjacente à bobina ${ }^{(\mathbf{5 0})}$.

Um dos únicos trabalhos que avaliaram se este acessório aumenta a sensibilidade do método na detecção de nódulos hepáticos foi realizado por Campeau et al., em $1995^{(\mathbf{5 0})}$. Os autores estudaram, prospectivamente, quatro sequiências, todas realizadas com bobina de corpo e com bobina de sinergia. As seqüências foram: SE-T2, GRE-T1, FSE-T2 em apnéia e EPI. Esses autores observaram que a sensibilidade na detecção de nódulos hepáticos foi maior (estatisticamente significante) em todas as seqüências realizadas com bobina de sinergia (Figura 3).

Outros trabalhos foram realizados para avaliar a utilidade da bobina de sinergia no estudo do abdome superior. Contudo, a maioria deles se deteve na análise qualitativa das imagens (demonstrando melhor 

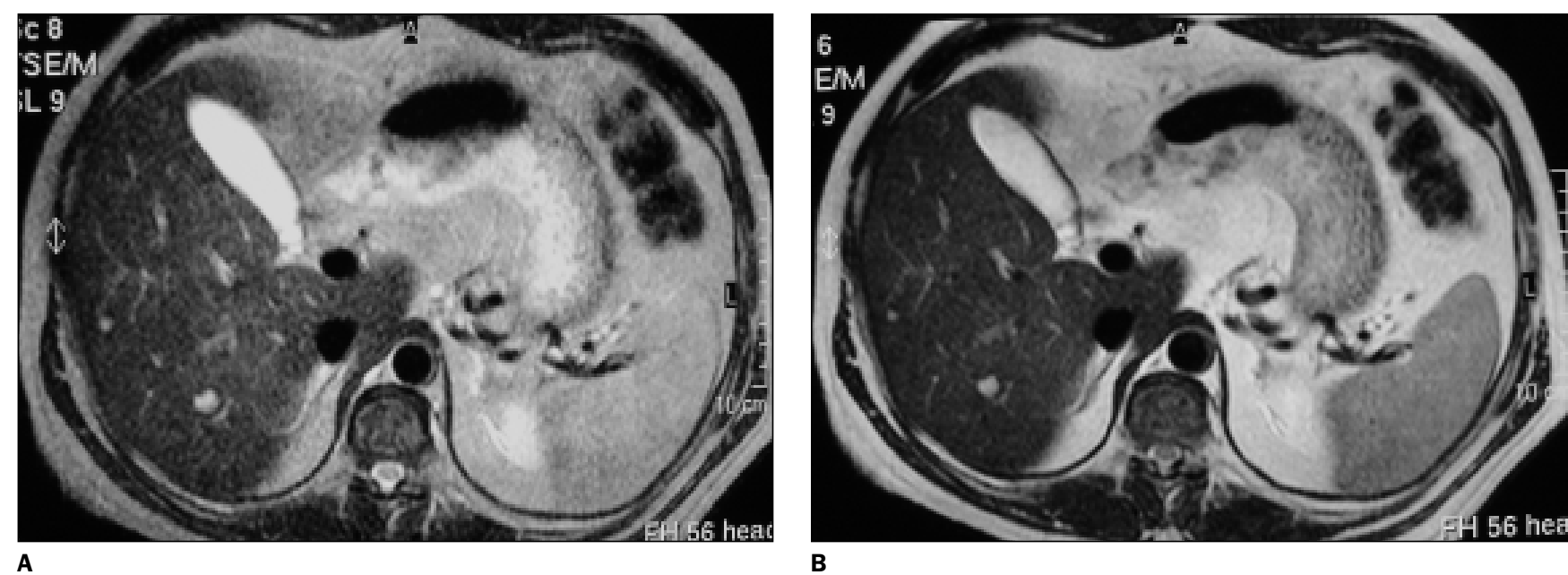

A

B

Figura 2. Seqüências TSE T2 (TR $=1.800$, TE $=90)$ com sincronizador respiratório, realizadas com $(\mathbf{A})$ e sem $(\mathbf{B})$ supressão de gordura. As duas seqüências demonstraram o mesmo número de lesões.

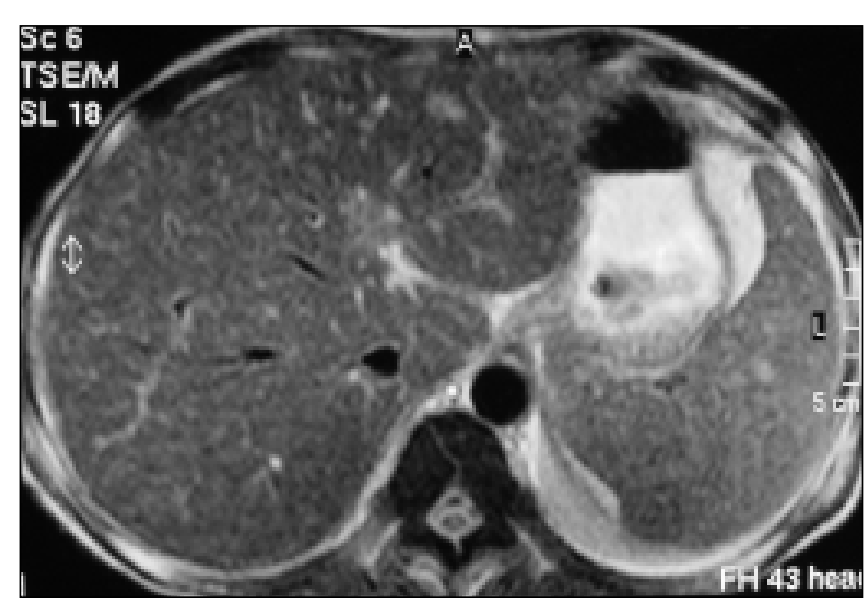

A

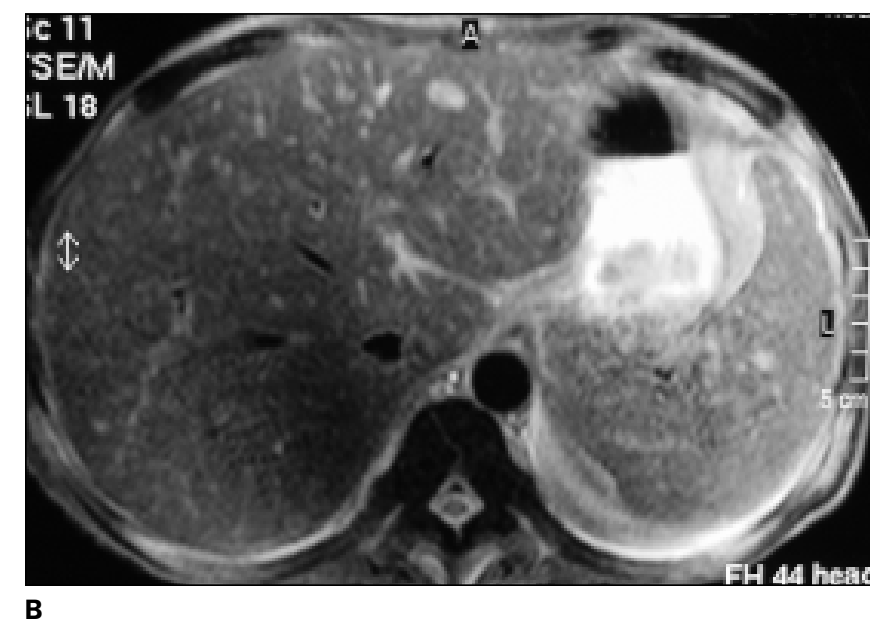

B

Figura 3. Seqüências TSE T2 (TR $=1.800$, TE $=90)$ realizadas com bobina de corpo $(\mathbf{A})$ e com bobina de sinergia $(\mathbf{B})$. Na seqüência realizada com bobina de sinergia as margens da lesão são mais bem definidas.

relação sinal/ruído e intensidade de sinal lesão/fígado superior à bobina de quadratura embutida no equipamento) e não pesquisaram efetivamente a sensibilidade da utilização deste acessório na detecção de lesões hepáticas focais ${ }^{(\mathbf{3 7}, \mathbf{4 3})}$.

\section{DISCUSSÃO}

As imagens ponderadas em T2 são comprovadamente úteis na detecção e caracterização de lesões hepáticas focais ${ }^{(\mathbf{1})}$.

Inicialmente, foram utilizadas com esta finalidade as sequiências SE. No entanto, estas seqüências requerem longo tempo de aquisição e freqüentemente apresentam imagens degradadas por artefatos de movimento, o que limita a sua utilização ${ }^{(4)}$. $\mathrm{Na}$ última década foram desenvolvidas se- qüências rápidas com a finalidade de reduzir o tempo de exame, reduzir o número de artefatos, melhorar a qualidade das imagens e, conseqüentemente, aumentar a acurácia na detecção de lesões hepáticas focais. Assim, surgiram as seqüências TSE (também conhecida como FSE ou RARE), HASTE, EPI e GRASE, acompanhadas ou não de técnicas de supressão de gordura, sincronização respiratória, apnéia e realizadas com bobina de corpo ou de superfície. A acurácia diagnóstica desses diferentes métodos de exame do fígado foi e tem sido motivo de constantes estudos e aprimoramento.

A partir dos estudos de Low et al., em 1993, vários outros trabalhos demonstraram as vantagens da utilização da seqüência TSE na detecção de nódulos hepáticos em relação à seqüência $\mathrm{SE}$ convencional ${ }^{(7-}$ 15). Atualmente, a técnica TSE/FSE é utilizada rotineiramente para a realização de imagens hepáticas ponderadas em T2, geralmente associada ao uso de sincronizador respiratório.

A acurácia da sequiência TSE foi também muitas vezes comparada à seqüência HASTE. Contudo, a este respeito ainda não existe um consenso de qual seqüência deveria ser realizada em um protocolo de avaliação do fígado. Para alguns autores, como Gaa et al., Tang et al. e Yamashita et al., a seqüência HASTE poderia ser utilizada como opção à sequiência TSE, com a vantagem do menor tempo de aquisição ${ }^{(15,18-23)}$. Porém, Kim et al. fazem parte do grupo de autores que contestam esta asserção, ao afirmarem que a seqüência 
HASTE apresenta sensibilidade menor na detecção de lesões hepáticas focais ${ }^{(\mathbf{2 6})}$.

A seqüência EPI é outro método de obtenção de imagem do fígado ponderada em T2 que surgiu em decorrência de avanços tecnológicos. Vários autores têm relatado a sua capacidade de detectar lesões hepáticas ${ }^{(14,15,21,27,28)}$. As imagens inicialmente utilizadas — "single-shot" EPI eram adquiridas em menor tempo, porém a qualidade da imagem era prejudicada principalmente devido aos inúmeros artefatos presentes, pelos efeitos de suscetibilidade magnética ${ }^{(14,15,21)}$. Yamakado et al. e Papanikolaou et al. tentaram demonstrar que estes efeitos são reduzidos quando se utiliza a seqüência "multi-shot" EPI, que requer maior tempo para ser adquirida, porém apresenta sensibilidade superponível à seqüência TSE na detecção de nódulos hepáticos ${ }^{(27,28)}$

Com a introdução da técnica em apnéia na avaliação do fígado, muitos trabalhos compararam-na com a sincronização respiratória para controle dos artefatos de respiração. Para Tang et al., Low et al. e outros autores, a utilização de sincronizadores respiratórios aumenta a sensibilidade das sequiências na detecção de lesões hepáticas focais ${ }^{(19,34-39)}$, porém uma desvantagem é o aumento no tempo de aquisição das imagens. Para Kim et al. e Earls et al. as seqüências realizadas em apnéia são as mais sensíveis ${ }^{(\mathbf{4 3}, 44)}$. Em vista disso, ainda há divergências a respeito da melhor técnica para controle desses artefatos.

Entre as técnicas básicas de supressão de gordura utilizadas na avaliação do abdome superior, todas apresentam vantagens e desvantagens. A mais utilizada nos estudos de sensibilidade na detecção de lesões hepáticas focais é a SPIR ou fat-sat. A maioria dos autores acredita que esta técnica aumenta a sensibilidade da sequiência na detecção de lesões hepáticas fo-

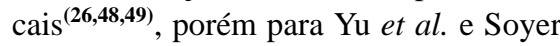
et al. parece não haver diferença estatisticamente significante ${ }^{(\mathbf{2 2 - 2 4 , 4 1 , 4 2 )} \text {. }}$

Quanto à utilização das bobinas de sinergia, está claro na literatura sua superioridade com relação à intensidade de sinal/ ruído na avaliação do abdome. Porém, elas foram pouco comparadas às bobinas de corpo quanto à sensibilidade na detecção de nódulos hepáticos. Apenas Campeau et al. realizaram um estudo com esta finalidade e obtiveram resultados satisfatórios com a bobina de sinergia ${ }^{(\mathbf{5 0})}$. No entanto, devido ao elevado custo deste acessório, existe a necessidade de estudos complementares a este respeito para justificar sua utilização de rotina nos exames de RM do fígado.

Outro recente avanço técnico tem sido descrito na literatura com o objetivo de estabelecer novas metodologias na avaliação de nódulos hepáticos, especialmente aqueles hipovascularizados. Trata-se da administração de meio de contraste paramagnético durante imagens ponderadas em T2. Embora os estudos ainda sejam iniciais, os resultados preliminares mostraram alta sensibilidade na detecção de nódulos sólidos hepáticos ${ }^{(\mathbf{5 1 , 5 2})}$.

Não analisamos aqui outras variáveis e parâmetros técnicos, como tempo de repetição (TR), tempo de eco (TE), fator turbo ("echo-train") e espessura do corte, entre outros, e que certamente influem na qualidade e resolução espacial da imagem de RM, por não ser o objetivo primordial deste trabalho. Também não avaliamos o uso de sequiências ponderadas em T1, com técnica gradiente-eco e injeção de contraste paramagnético, notoriamente úteis na detecção de tumores hepáticos hipervascularizados, pois focalizamos nossa atenção nas imagens ponderadas em T2.

Em resumo, após revisão bibliográfica, percebemos que não existe consenso na técnica de aquisição de imagens ponderadas em T2 para estudos do fígado, principalmente no que se refere ao uso de apnéia, sincronização respiratória e supressão de gordura. A eficácia destes recursos parece depender do tipo de equipamento utilizado e da experiência do radiologista. No entanto, é importante observar que é indispensável desenhar um protocolo eficiente e otimizado para se obter os melhores resultados, através da RM, cuja utilização no estudo do abdome superior tem crescido notavelmente. Novos estudos, randomizados, prospectivos e com estrito controle das diversas variáveis serão ainda necessários para estabelecer quais as técnicas e seqüências indispensáveis para a avaliação hepática, associando alta sensibilidade na detecção de lesões focais com tempos de aquisição aceitáveis.

\section{REFERÊNCIAS}

1. Ito K, Mitchell DG, Outwater EK, Szklaruk J, Sadek AG. Hepatic lesions: discrimination of nonsolid, benign lesions from solid, malignant lesions with heavily T2-weighted fast spin-echo MR imaging. Radiology 1997;204:729-37.

2. Doyle FH, Pennock JM, Banks LM, et al. Nuclear magnetic resonance imaging of the liver: initial experience. AJR 1982;138:193-200.

3. Stark DD, Wittenberg J, Middleton MS, Ferrucci JT Jr. Liver metastases: detection by phase-contrast MR imaging. Radiology 1986;158:327-32.

4. Reinig JW, Dwyer AJ, Miller DL, Frank JA, Adams GW, Chang AE. Liver metastases: detection with MR imaging at 0.5 and 1.5 T. Radiology $1989 ; 170$ (1 Pt 1):149-53.

5. Steinberg HV, Alarcon JJ, Bernardino ME. Focal hepatic lesions: comparative MR imaging at 0.5 and 1.5 T. Radiology 1990;174:153-6.

6. Low RN, Francis IR, Sigeti JS, Foo TKF. Abdominal MR imaging: comparison of T2-weighted fast and conventional spin-echo, and contrast-enhanced fast multiplanar spoiled gradient-recalled imaging. Radiology 1993;186:803-11.

7. Kreft B, Layer G, Spiller L, et al. Evaluation of rapid gradient-echo and turbo-spin-echo sequences in MRT of focal liver lesions using 0.5 Tesla. Rofo Fortschr Geb Rontgenstr Neuen Bildgeb Verfahr 1994;161:512-8

8. Giovagnoni A, Ercolani P, Terilli F, Ferrara C, Paci E, Santino P. Turbo-spin-echo sequences in focal liver diseases. Radiol Med (Torino) 1994;87:63642.

9. Giovagnoni A, Paci E, Ercolani P, Kiefer B, Santino P, Piga A. MR imaging of focal liver lesions: comparison between turbo spin-echo and conventional spin-echo pulse sequences. MAGMA 1995;3:14350.

10. Manfredi R, Maresca G, Tartaglione T, Scarano E, Marano P. T2-dependent sequences in the study of hepatic focal lesions: comparison of the conventional spin echo sequence and the $0.5 \mathrm{~T}$ fast spin echo. Radiol Med (Torino) 1996;92:236-40.

11. Baudouin CJ, Ward J, Ridgway JP, Robinson PJ. A comparison of fast and conventional T2 weighted spin-echo sequences in the detection of focal liver lesions at 1.0 T. Clin Radiol 1996;51:769-74.

12. Reimer P, Rummeny EJ, Wissing M, Bongartz GM, Schuierer G, Peters PE. Hepatic MR imaging: comparison of RARE derived sequences with conventional sequences for detection and characterization of focal liver lesions. Abdom Imaging 1996;21: 427-32.

13. Keogan MT, Spritzer CE, Paulson EK, et al. Liver MR imaging: comparison of respiratory triggered fast spin echo with T2-weighted spin-echo and inversion recovery. Abdom Imaging 1996;21:433-9.

14. Gaa J, Hatabu H, Jenkins RL, Finn JP, Edelman RR. Liver masses: replacement of conventional T2weighted spin-echo MR imaging with breath-hold MR imaging. Radiology 1996;200:459-64.

15. Gaa J, Fischer H, Laub G, Georgi M. Breath-hold MR imaging of focal liver lesions: comparison of fast and ultrasound techniques. Eur Radiol 1996; 6:838-43.

16. Rydberg JN, Lomas DJ, Coakley KJ, Hough DM, Ehman RL, Riederer SJ. Comparison of breath-hold fast spin-echo and conventional spin-echo pulse sequences for T2-weighted MR imaging of liver lesions. Radiology 1995;194:431-7. 
17. Helmberger T, Holzknecht N, Lackerbauer CA, et $a l$. Phased-array superficial coil and breath holding technique in MRI of the liver. Comparison of conventional spin echo sequences with rapid fat suppressing gradient echo and turbo-spin sequences. Radiologe 1995;35:919-24.

18. Van Hoe L, Bosmans H, Aerts P, et al. Focal liver lesions: fast T2-weighted MR imaging with halfFourier rapid acquisition with relaxation enhancement. Radiology 1996;201:817-23.

19. Tang Y, Yamashita Y, Namimoto T, Abe Y, Takahashi M. Liver T2-weighted MR imaging: comparison of fast and conventional half-Fourier singleshot turbo spin-echo, breath-hold turbo spin-echo, and respiratory triggered turbo spin-echo sequences. Radiology 1997;203:766-72.

20. Tang Y, Yamashita Y, Namimoto T, Takahashi M. Characterization of focal liver lesions with halfFourier acquisition single-shot turbo-spin-echo (HASTE) and inversion recovery (IR)-HASTE sequences. J Magn Reson Imaging 1998;8:438-45.

21. Yamashita Y, Tang Y, Namimoto T, Mitsuzaki K, Takahashi M. MR imaging of the liver: comparison between single-shot echo-planar and half-Fourier rapid acquisition with relaxation enhancement sequences. Radiology 1998;207:331-7.

22. Brinkmann G, Musiolik I, Kuhn B, et al. Ultra-rapid T2-weighted MR imaging during suspended respiration for the examination of focal lesions of the liver: a comparison of TSE, HASTE and HASTESTIR sequences using a CP body array coil. Rofo Fortschr Geb Rontgenstr Neuen Bildgeb Verfahr 1998;168:330-6.

23. Yu JS, Kim KW, Kim YH, Jeong EK, Chien D. Comparison of multishot turbo spin echo and HASTE sequences for T2-weighted MRI of liver lesions. J Magn Reson Imaging 1998;8:1079-84.

24. Lee MG, Jeong YK, Kim JC, et al. Fast T2-weighted liver MR imaging: comparison among breathhold turbo-spin-echo, HASTE, and inversion recovery (IR) HASTE sequences. Abdom Imaging 2000; 25:93-9.

25. Helmberger TK, Schroder J, Holzknecht N, et al. T2-weighted breathold imaging of the liver: a quantitative and qualitative comparison of fast spin echo and half-Fourier single shot fast spin echo imaging. MAGMA 1999;9:42-51.

26. Kim TK, Lee HJ, Jang HJ, Kim AY, Han JK, Choi BI. T2-weighted breath-hold MRI of the liver at 1.0 $\mathrm{T}$ : comparison of turbo spin-echo and HASTE sequences with and without fat suppression. J Magn Reson Imaging 1998;8:1213-8.

27. Yamakado K, Sakuma H, Murashima S, Nakatsuka A, Matsumura K, Takeda K. Comparison of breathhold multishot echo-planar and respiratory triggered fast-spin-echo sequences for T2-weighted MRI of liver lesions. J Magn Reson Imaging 1998; 8:432-7.

28. Papanikolaou N, Moulopoulos LA, Gouliamos A, Ispanopoulou S, Vlahos L. Comparison of dual spin echo echo planar imaging (SE-EPI), turbo spin echo with fat suppression and conventional dual spin echo sequences for $\mathrm{T}(2)$-weighted MR imaging of focal liver lesions. Magn Reson Imaging 2000;18: 715-9.

29. Feinberg DA, Oshio K. GRASE (gradient- and spinecho) MR imaging: a new fast clinical imaging technique. Radiology 1991;181:597-602.

30. Jung G, Krahe T, Kugel H, et al. Prospective comparison of fast SE and GRASE sequences and echo planar imaging with conventional SE sequences in the detection of focal liver lesions at 1.0 T. J Comput Assist Tomogr 1997;21:341-7.

31. Karantanas AH, Papanikolaou N. T2-weighted magnetic resonance imaging of the liver: comparison of fat-suppressed GRASE with conventional spin echo, fat-suppressed turbo spin echo, and gradient echo at 1.0 T. Abdom Imaging 2001;26:13945.

32. Pijl MEJ, Wasser NM, van Meerten EP, et al. Comparison of inversion-recovery gradient- and spinecho and fast spin-echo techniques in the detection and characterization of liver lesions. Radiology 1998;209:427-34.

33. Mirowitz SA. MR imaging artifacts. Challenges and solutions. Magn Reson Imaging Clin N Am 1999; 7:717-32.

34. Low RN, Alzate GD, Shimakawa A. Motion suppression in MR imaging of the liver: comparison of respiratory-triggered and nontriggered fast spinecho sequences. AJR 1997;168:225-31.

35. Kanematsu M, Hoshi H, Murakami T, et al. Focal hepatic lesion detection: comparison of four T2weighted MR imaging pulse sequences. Radiology 1998;206:167-75.

36. Kanematsu M, Hoshi H, Itoh K, et al. Focal hepatic lesion detection: comparison of four fat-suppressed T2- weighted MR imaging pulse sequences. Radiology 1999;211:363-71.

37. Namimoto T, Yamashita Y, Mitsuzaki K, Takahashi $M$. The value of respiratory triggered $\mathrm{T} 2$-weighted turbo spin-echo imaging of the liver using a phased array coil. J Magn Reson Imaging 1998;8:655-62.

38. Hori M, Murakami T, Kim T, et al. Single breathhold T2-weighted MR imaging of the liver: value of single-shot fast spin-echo and multishot spinecho echoplanar imaging. AJR 2000;174:142331.

39. Pawluk RS, Borrello JA, Brown JJ, McFarland EG, Mirowitz SA, Tsao LY. A prospective assessment of breath-hold fast spin echo and inversion recovery fast spin echo techniques for detection and characterization of focal hepatic lesions. Magn Reson Imaging 2000;18:543-51.

40. Carpenter KD, Macaulay SE, Schulte SJ, et al. MR of focal liver lesions: comparison of breath-hold and non-breath-hold hybrid RARE and conventional spin-echo T2-weighted pulse sequences. J Magn Reson Imaging 1996;6:596-602.
41. Soyer P, de Givry SC, Lenormand S, Somveille E, Scherrer A. Detection of focal hepatic lesions with MR imaging: prospective comparison of T2weighted fast spin-echo with and without fat suppression, T2-weighted breath-hold fast spin-echo, and gadolinium chelate-enhanced 3D gradient-recalled imaging. AJR 1996;166:1115-21.

42. Soyer P, Gouhiri M, Rondeau Y, Spelle L, Mosnier $\mathrm{H}$, Scherrer A. Non-breath-hold fast spin-echo versus breath-hold fast spin-echo and spoiled gradientrecalled echo MR imaging in the detection of hepatic tumors: correlation with surgical findings. AJR 1997;168:1199-204.

43. Kim TK, Wang WC, Han JK, Cho SG, Choi BI. T2weighted MR imaging for hepatic hemangiomas: comparison of breath-hold and non-breath-hold turbo spin-echo pulse sequences with phased-array multicoil. Abdom Imaging 1998;23:422-6.

44. Earls JP, Rofsky NM, DeCorato DR, Krinsky GA, Weinreb JC. Echo-train STIR MRI of the liver: comparison of breath-hold and non-breath-hold imaging strategies. J Magn Reson Imaging 1999; 9:87-92.

45. Delfaut EM, Beltran J, Johnson G, Rousseau J, Marchandise X, Cotten A. Fat suppression in MR imaging: techniques and pitfalls. Radiographics 1999;19:373-82.

46. Bydder GM, Steiner RE, Blumgart LH, Khenia S, Young IR. MR imaging of the liver using short T1 inversion recovery sequences. J Comput Assist Tomogr 1985;9:1084-9.

47. Schwartz LH, Seltzer SE, Tempany CMC, et al. Prospective comparison of T2-weighted fast spinecho, with and without fat suppression, and conventional spin-echo pulse sequences in the upper abdomen. Radiology 1993;189:411-6.

48. Lu DS, Saini S, Hahn PF, et al. T2-weighted MR imaging of the upper part of the abdomen: should fat suppression be used routinely? AJR 1994;162: 1095-100

49. Kreft B, Layer G, Kuhl C, Sommer T, Gieseke J, Schild H. Turbo-spin-echo sequences with selective fat suppression (SPIR) in the MRI of focal liver lesions at 0.5 Tesla. Rofo Fortschr Geb Rontgenstr Neuen Bildgeb Verfahr 1995;163:411-6.

50. Campeau NG, Johnson CD, Felmlee JP, et al. MR imaging of the abdomen with a phased-array multicoil: prospective clinical evaluation. Radiology 1995;195:769-76.

51. Jeong YY, Mitchell DG, Holland GA. Liver lesion conspicuity: T2-weighted breath-hold fast spinecho MR imaging before and after gadolinium enhancement - initial experience. Radiology 2001; 219:455-60

52. Murakami T, Kim T, Hori M, et al. Multishot echoplanar imaging with gadopentetate dimeglumine. Preliminary study of efficacy for detection of hypovascular metastatic liver tumors. Acta Radiol 2000;41:263-8. 